Brandl, Werner

\title{
Bausteine und Baustelle einer Didaktik subjektorientierten Lernens und
}

\section{Lehrens}

Haushalt in Bildung \& Forschung 3 (2014) 3, S. 33-53

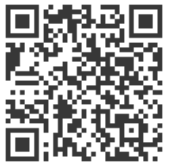

Quellenangabe/ Reference:

Brandl, Werner: Bausteine und Baustelle einer Didaktik subjektorientierten Lernens und Lehrens - In: Haushalt in Bildung \& Forschung 3 (2014) 3, S. 33-53 - URN: urn:nbn:de:0111-pedocs-204259 - DOI: 10.25656/01:20425

https://nbn-resolving.org/urn:nbn:de:0111-pedocs-204259

https://doi.org/10.25656/01:20425

in Kooperation mit / in cooperation with:

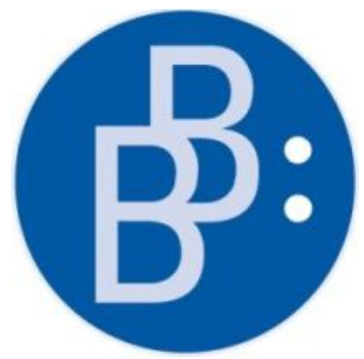

https://www.budrich.de

\section{Nutzungsbedingungen}

Gewährt wird ein nicht exklusives, nicht übertragbares, persönliches und beschränktes Recht auf Nutzung dieses Dokuments. Dieses Dokument is ausschließlich für den persönlichen, nicht-kommerziellen Gebrauch bestimmt. Die Nutzung stellt keine Übertragung des Eigentumsrechts an diesem Dokument dar und gilt vorbehaltlich der folgenden Einschränkungen: Auf sämtlichen Kopien dieses Dokuments müssen alle Urheberrechtshinweise und sonstigen Hinweise auf gesetzlichen Schutz beibehalten werden. Sie dürfen dieses Dokument nicht in irgendeiner Weise abändern, noch dürfen Sie dieses Dokument für öffentliche oder kommerzielle Zwecke vervielfältigen, öffentlich ausstellen, aufführen, vertreiben oder anderweitig nutzen.

Mit der Verwendung dieses Dokuments erkennen Sie die Nutzungsbedingungen an.

\section{Terms of use}

We grant a non-exclusive, non-transferable, individual and limited right to using this document.

This document is solely intended for your personal, non-commercial use. Use of this document does not include any transfer of property rights and it is conditional to the following limitations: All of the copies of this documents mus retain all copyright information and other information regarding legal protection. You are not allowed to alter this document in any way, to copy it for public or commercial purposes, to exhibit the document in public, to perform, distribute or otherwise use the document in public.

By using this particular document, you accept the above-stated conditions of use.

\section{Kontakt / Contact:}

\section{peDOCS}

DIPF | Leibniz-Institut für Bildungsforschung und Bildungsinformation Informationszentrum (IZ) Bildung

E-Mail: pedocs@dipf.de

Internet: www.pedocs.de

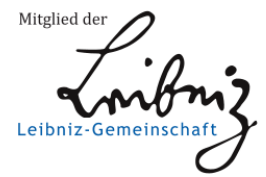


3. Jahrgang Heft 3

2014

는

๓)

$\bullet$

(1)

$\frac{1}{\bar{E}}$

0

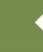

(1)

(

+

ᄃ

믕

은

논

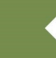

๕

(1)

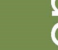

$\varepsilon$

ह

$\frac{\mathcal{B}}{\mathrm{N}}$

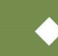

$+$

$\stackrel{\$}{ٍ}$

$\bullet$

$\frac{1}{\Phi}$
$\frac{c}{0}$
$\frac{5}{5}$
d
0

\section{Haushalt in Forschung}

\section{Subjektorientierung in der}

\section{Ernährungs- und Verbraucherbildung}


Inhaltsverzeichnis |

Silke Bartsch \& Claudia Wespi

Editorial. 2

Silke Bartsch \& Barbara Methfessel

Der „subjektive Faktor“. Bildung in einem lebensweltorientierten Fach 3

Werner Brandl

Bausteine und Baustelle einer Didaktik subjektorientierten Lernens und Lehrens... 33

Claudia Wespi \& Corinne Senn Keller

Subjektorientiertes Lernen und Lehren in einer kompetenzorientierten

Unterrichtskonzeption

Käthi Theiler-Scherrer \& Corinne Senn Keller

Simulationsspiel als subjektorientierte Konsumbildung 75

Barbara Methfessel \& Bärbel Schön

Biographisch orientierte Lehr-Lernprozesse als „Brücke“ zwischen

lebensweltlichen Erfahrungen und systematischen Lehr-Lernprozessen -

Erfahrungen aus einem Lehr-Forschungsprojekt.

Nicola Kluß

Ernährungswissen und -handeln am Beispiel von Vollkorn - ein Lehr-

Forschungsprojekt.

Werner Brandl

Rezension: Didaktische Prinzipien.

Melanie Lukas

Rezension: Wesen und Wege nachhaltigen Konsums. 
Werner Brandl

\section{Bausteine und Baustelle einer Didaktik subjektorientierten Lernens und Lehrens}

Didaktik - kurz gefasst als die Wissenschaft vom Lernen und Lehren - ist nicht allein mit Begriffen „einheimischer“ Provenienz zu begründen - die Diskussion über die theoretische Tragweite und praktische Belastbarkeit einer Didaktik subjektorientierten Lernens und Lehrens in der Ernährungs- und Verbraucherbildung (EVB) verweist notwendigerweise auf eine lern- und lehrtheoretische Fundierung.

Schlüsselwörter: Didaktik, subjektorientiertes Lernen und Lehren, adaptive Lehrkompetenz

\section{Was ist das Subjekt?}

Schon Kant (1789) kam in der Beantwortung der eigentlich genuin anthropologischen Fragestellung „Was ist der Mensch?“ nicht umhin, auf Basis einer „Anthropologie in pragmatischer Hinsicht" sich auf spezifische Aspekte des Menschseins zu beziehen: „Der Mensch ist durch seine Vernunft bestimmt, in einer Gesellschaft mit Menschen zu sein, und in ihr sich durch Kunst und Wissenschaft zu kultivieren, zu zivilisieren und zu moralisieren" (S. 324).

Mit einem Blick auf die wissenschaftliche Betrachtung des Menschen wird schon evident, dass in den diversen Natur- und Geisteswissenschaften und dort je nach Standpunkt je spezifische Aspekte des Menschseins im Zentrum der theoretischen Fassung stehen. Der evolutionsbiologischen Systematik nach ist die Einordnung als homo sapiens und Abgrenzung von anderen Lebewesen noch recht einfach und einsichtig, in der Einschätzung als „Primus inter Primates“ beginnen dann allerdings bereits die differenten Blickwinkel mit einem spezifischen Menschenbild, das je nach Zugehörigkeit zu einer historischen Epoche, einem Kulturkreis, einer Religion, politischen Verfassung, sozio-ökonomischen Status etc. sehr unterschiedlich und vielfältig in der Interpretation als soziales Wesen ausfallen kann: Die Vorstellungen vom Ebenbild des Schöpfers bis zum Sündenfall, von der Krone der Schöpfung bis zum GAU der Weltgeschichte, vom Untertan bis zur Ich-AG, vom Wesen mit freiem Willen und dem Gegenteil, vom homo oeconomicus zum homo educandus usw. usf. waren und sind anthropogene Konstruktionen einer anthropologischen Natur des Menschen - allein die Blickwinkel auf und Begründungen dafür wechseln (vgl. Abb. 1)! 


\section{Didaktik subjektorientierten Lernens und Lehrens}

Auffällig ist allerdings auch, dass alltagssprachlich wie im wissenschaftlichen Sprachgebrauch neben der Begriffsbestimmung ex termini unmittelbar eine Konnotation ex negativo existiert: Subjekt vs. Objekt, Individuum vs. Gruppe (o. Ä.), Person: natürliche vs. juristische. Ob gewollt oder nicht bzw. bewusst oder unbewusst, erfährt der jeweilige Terminus auf einer Plus-Minus-Skala eine eindeutige Einordnung, der man (so es überhaupt denkbar ist) den gegenteiligen Pol argumentativ nur sehr eingeschränkt bzw. gar nicht zuordnen kann: Wer möchte schon, dass der Schüler ausschließlich als Objekt betrachtet, auf ein Gruppenmitglied im Klassenverband reduziert wird und nicht als Person behandelt wird? - die Würde des Menschen ist sowieso unantastbar!

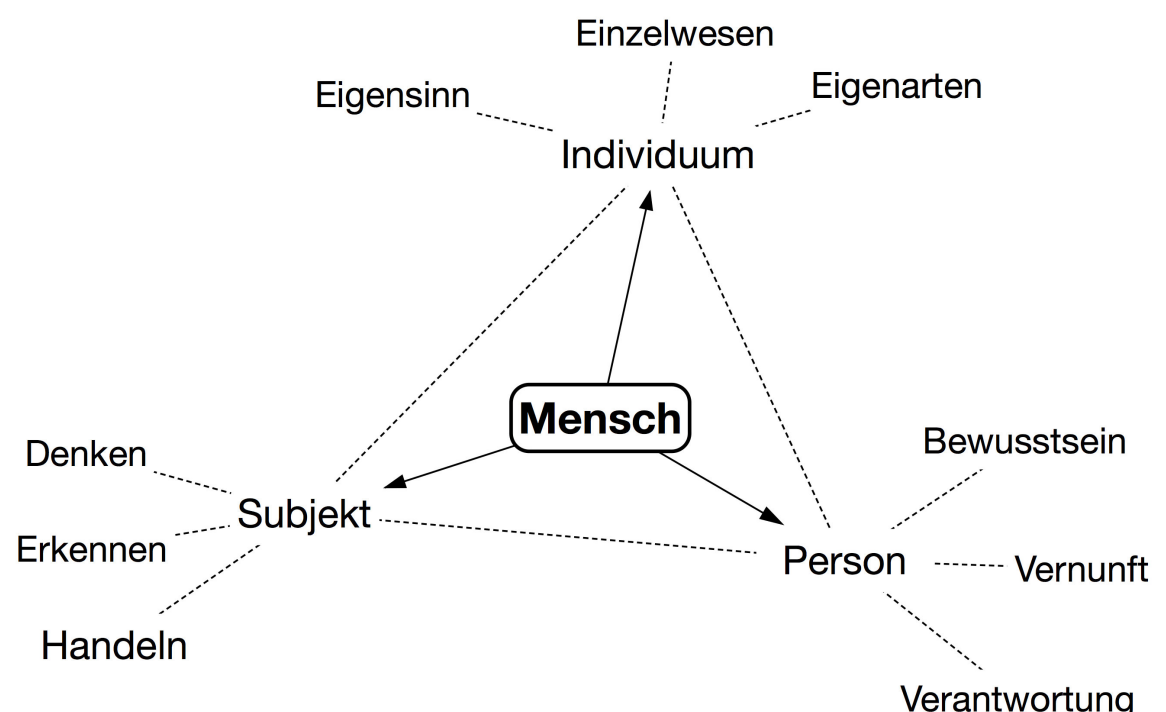

Abb. 1: Der Mensch - und seine Facetten (Quelle: Eigene Darstellung)

Die Konzeption einer subjektorientierten Didaktik kommt insofern auch nicht umhin, dem unmittelbar positiv konnotierten Begriff der Subjektorientierung nicht ,blindlings' zu folgen und ebenfalls nicht über dem Subjektbegriff die für einen alltags- und lebensweltorientierten Ansatz (vgl. Methfessel \& Schlegel-Matthies, 2014) geradezu essenziellen anderen Facetten zu vernachlässigen. Die Komplexität des Gegenstandsbereiches der Ernährungs- und Verbraucherbildung und der Lebensumstände der Akteure bzw. Adressaten machen es unumgänglich, die spezifischen Möglichkeiten und Notwendigkeiten von Person, Subjekt und Individuum bezüglich der

- biografischen Lebens-Geschichte(n)

- aktuellen Lebens-Welt(en) 


\section{Didaktik subjektorientierten Lernens und Lehrens}

- perspektivischen Lebens-Konzept(en)

- tatsächlichen Lebens-Praxen

einzubringen und abzubilden - und die strukturellen Aspekte darüber nicht zu vernachlässigen, die sich als Spannungsfeld zwischen (individuellem) Verhalten und den (sozio-ökonomischen) Verhältnissen - zwar knapp, aber stimmig - zusammenfassen lässt: „Die alltägliche Lebensführung ist zwar eine individuelle Leistung, die aber durch die Rahmenbedingungen der jeweiligen sozialen Gemeinschaft/Gruppe beeinflusst, wenn nicht sogar gesteuert wird" (Methfessel \& Schlegel-Matthies, 2014, S. 34). Die unterschiedliche, potenziell gegensätzliche Betrachtung aus der Innen- und/oder Außenperspektive (vgl. Abb. 2) setzt sich in ebensolchen Differenzen fort, mit der mittels deskriptiv-explanativen, präskriptiven und normativen $\mathrm{Zu}-$ gängen gleiche oder ähnliche Sachverhalte und Beziehungen thematisiert werden: Das Verbraucherhandeln stellt sich in der Beschreibung und Begründung aus der Innenperspektive oftmals anders als in der Erklärung aus der Außenperspektive dar.

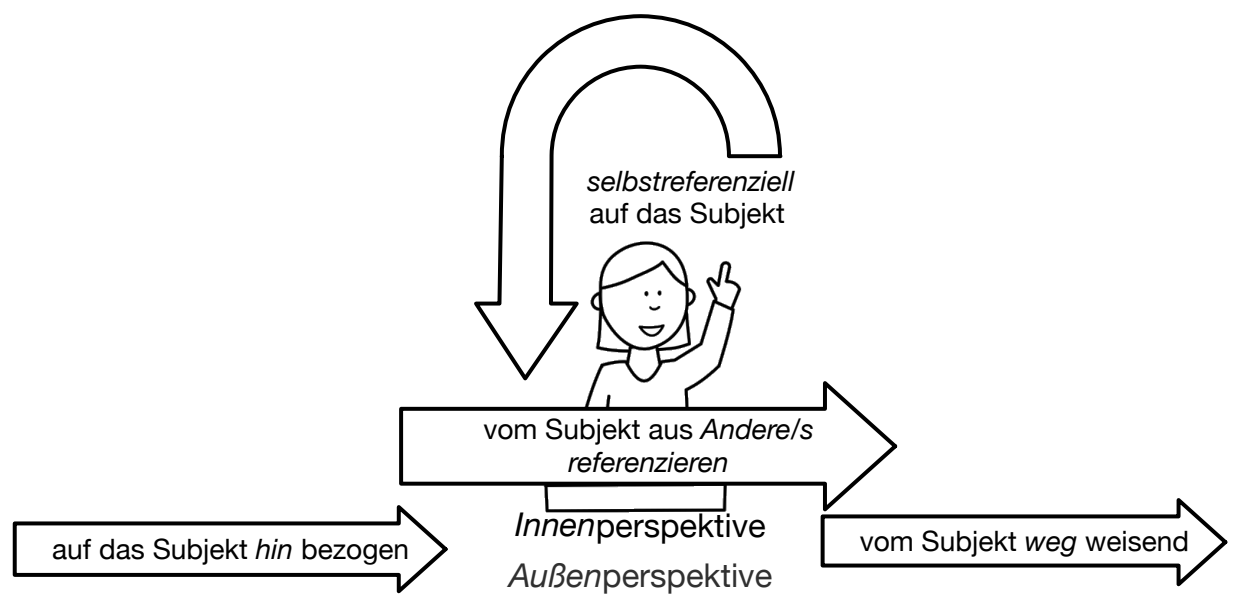

Abb. 2: Das Subjekt in der Innen-/Außenperspektive (Quelle: Eigene Darstellung)

Ohne allzu sehr in die philosophischen, soziologischen, ökonomischen, psychologischen und sonstigen Ableitungen und Begründungen für deren begriffliche Verwendung einzusteigen (vgl. dazu Bräuer, 2003; Keupp, 2012; Lüdtke \& Matsuzaki, 2011), bleibt festzuhalten, dass mit dem Individuum nicht nur dessen (physische wie psychische) Eigenheiten als Einzelwesen gemeint sind, sondern ebenso die für Lernprozesse förderlichen wie hinderlichen eigenwilligen und eigensinnigen $\mathrm{Zu}$ gänge. Als subjektives Denken, Erkennen und Handeln gefasst, konstituieren diese im Kern das, was das Subjekt als Akteur im Unterschied zu ihm als Adressat von Lernansprüchen ausmacht. Und als Person betrachtet, ist es diejenige Instanz, die mit Bewusstsein, Vernunft und Willen ausgestattet, die Verantwortung für das eigene Handeln trägt - und das nicht nur beim Lernen! 


\section{Didaktik subjektorientierten Lernens und Lehrens}

\section{Lernen subjektorientiert verstehen...}

Eigentlich eine Selbstverständlichkeit: Wer, außer den Lernenden als Subjekte, kann, will, soll und muss lernen? „Verstehen und Lernen als Aneignung von bislang nicht Verstandenem, bislang nicht Gewusstem und nicht Gekonntem stellen unverwechselbare Subjektleistungen dar" (Meueler, 2011, S. 974). Und dennoch ist die lerntheoretische und didaktische Diskussion über den geeigneten Weg und dazu passende Maßnahmen nicht abgeschlossen - wird vielmehr in letzter Zeit wieder vehement geführt. Ausgangspunkt bleibt der Disput über das jeweilig zugrundeliegende Menschenbild - nicht unbedingt als rein anthropologische Frage nach der Erziehungsbedürftigkeit und Lernfähigkeit des Menschen, sondern in Bezug auf die Bildungsziele und Gestaltung von entsprechenden Lernumgebungen.

Die Frage nach dem „,subjektiven Faktor in einer lebensweltbezogenen Bildung“ (Methfessel und Bartsch i. d. H) und die Diskussion über eine Didaktik subjektorientierten Lernens und Lehrens (vgl. Tulodziecki, 2002; Bartsch, 2008; 2012), thematisieren erneut (und mit etwas anderem) Blick die bereits im REVIS-Projekt explizierten Bildungsziele und das salutogenetisch orientierte und kompetenzorientierte Lernen als zentrale didaktische Prinzipen (neben dem lebensbegleitenden Lernen).

\subsection{Grundlegende psychologische Bedürfnisse}

Eine dezidiert alltags- und lebensweltbezogene Bildung, wie sie die Ernährungs- und Verbraucherbildung darstellt, ist in Bezug auf die "Subjekte“ in einer Weise befasst, die diese in zweifacher Hinsicht tangiert: Zum einen sind sie als unmittelbar in dieser Lebenswelt verankerte Akteure betroffen und zum anderen werden ihnen als Adressaten extern - der eigenen Lebenswelt entnommene - Lerninhalte an sie zur Bearbeitung herangetragen, die sie (hoffentlich) mit Surplus wieder zurückerhalten!

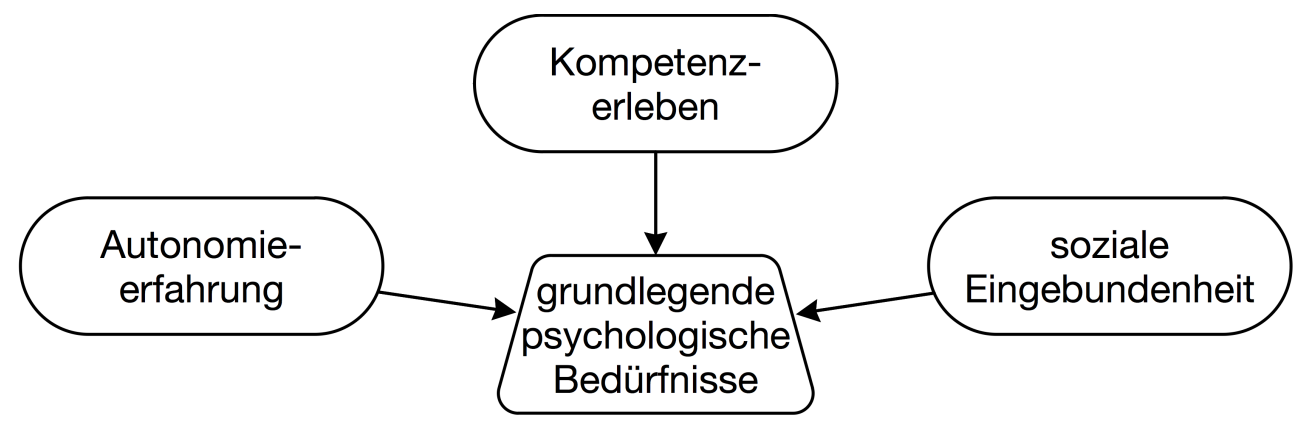

Abb. 3: „Basic human needs“ nach Deci \& Ryan (2000) (Quelle: Eigene Darstellung)

Mit den grundlegenden psychologischen Bedürfnissen („Basic human needs“; vgl. Deci \& Ryan, 2000) werden nicht nur die „Basics“ für die gelingende Gestaltung von Biografie und Lebenslauf präsentiert, sondern darüber hinaus diese ,individuellen 


\section{Didaktik subjektorientierten Lernens und Lehrens}

Lebensinteressen als lerntheoretische Grundkategorie“ (Krapp, 2004) postuliert und als damit grundlegende Bedingung für erfolgreiche Lernprozesse formuliert. Im Rahmen der Selbstbestimmungstheorie der Motivation spielen die Aspekte des Erlebens von Kompetenz, der Erfahrung von Autonomie, aber auch die Notwendigkeit sozialer Eingebundenheit (vgl. Abb. 3) eine erhebliche Rolle.

Für eine Ernährungs- und Verbraucherbildung mit Bezug auf die alltägliche Lebensführung und -gestaltung sind diese grundlegenden Bedürfnisse nicht nur Bedürfnisse im Sinne einer Defizitbeseitigung, sondern zentrale Ausgangs- Prozessund Zieldimensionen pädagogischen Einwirkens: Ob und wie sich Verbraucher z. B. in ihrem Ernährungsverhalten als kompetent betrachten, als autonom handelnd sehen und sozial akzeptiert fühlen - und wenn nein, warum und was dem entgegensteht ist nicht nur auf der deskriptiven Ebene als status quo interessant, sondern bedeutet auf der präskriptiv-normativen Ebene Entscheidungen über die Ziel- und Prozessvariablen für als notwendig erachtete pädagogische Interventionen.

\subsection{Salutogenese - didaktisch betrachtet}

Im REVIS-Projekt wurde das salutogenetisch orientierte Lernen als ein zentrales didaktisches Prinzip (neben dem kompetenzorientierten und lebensbegleitenden Lernen; vgl. Methfessel, 2007; 2008) für das Lernen in der EVB ausformuliert. Antonovskys Überlegungen, dass das Kohärenzgefühl mit den Komponenten Verstehbarkeit, Handhabbarkeit und Bedeutsamkeit/Sinnhaftigkeit (siehe: Glossar [www.hibifo.de/glossar.html]) unschätzbare Ressourcen für die Gesunderhaltung (auch unter widrigen Situationen) darstellen, wurden für die Ernährungs- und Verbraucherbildung adaptiert.

Mit dem Kohärenzsinn bzw. -gefühl beschreibt Antonovsky „eine globale Orientierung, die das Ausmaß ausdrückt, in dem jemand ein durchdringendes, überdauerndes und dennoch dynamisches Gefühl des Vertrauens hat,

- dass erstens die Anforderungen aus der inneren oder äußeren Erfahrenswelt im Verlauf des Lebens strukturiert, vorhersagbar und erklärbar sind und

- dass zweitens die Ressourcen verfügbar sind, die nötig sind, um den Anforderungen gerecht zu werden.

- Und drittens, dass diese Anforderungen Herausforderungen sind, die Investition und Engagement verdienen“ (Antonovsky zit. nach Bengel et al., 2001, S. 30). 


\section{Didaktik subjektorientierten Lernens und Lehrens}

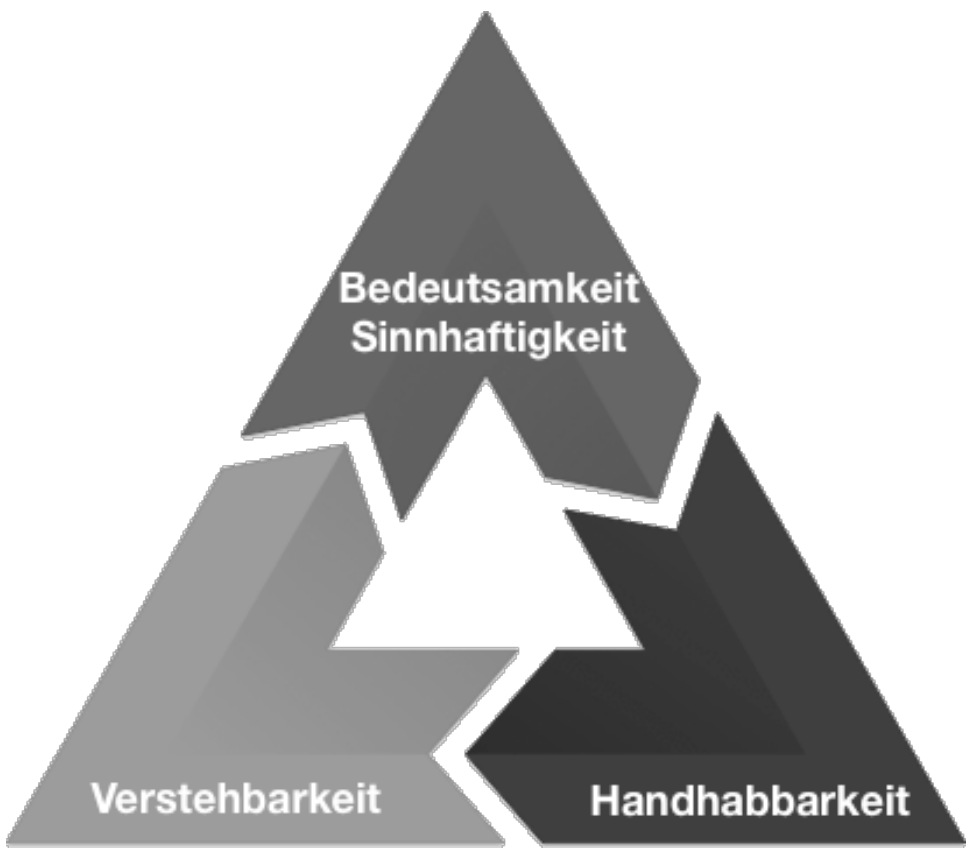

Abb. 4: Kohärenzsinn und -gefühl - nicht nur salutogenetisch, auch didaktisch! (Quelle: Eigene Abbildung)

Der salutogenetische Ansatz mit den Ankern der Verstehbarkeit, Handhabbarkeit und Bedeutsamkeit/Sinnhaftigkeit gibt auch unter didaktischen Gesichtspunkten einen Blick auf Unterricht und Erziehung in der Ernährungs- und Verbraucherbildung frei, der zunächst unstatthaft erscheint, auf einen zweiten Blick hingegen Sinn macht: Die Lerngegenstände und - was das Wesentliche ist - die Lernergebnisse sind ganz unmittelbar als Handlungsoptionen in den Lebenskontext eingebunden, der mit den Aspekten der Verstehbarkeit, Handhabbarkeit und Bedeutsamkeit/Sinnhaftigkeit eine Überführung in tatsächliches Ernährungs-, Gesundheits- und Verbraucherhandeln befördern oder auch behindern kann.

\subsection{Kompetenzen}

Mit dem Kompetenzbegriff wird im Zusammenhang mit Lernen und Lehren sehr gehadert - insbesondere von Seiten der Allgemeinen Pädagogik und Didaktik. Allerdings weniger wegen der begrifflichen Fassung von Kompetenz als solcher, sondern vielmehr als ein Angriff auf die genuine Zuständigkeit für die Bildung; hier sieht man den Bildungsbegriff in einer Reduktion insofern ausgehöhlt, als man mit dem Erwerb und der Entwicklung von Kompetenzen eine reine Funktionalität in Wirtschaft und Gesellschaft unterstellt, die den individuellen Bildungsanspruch konterkariert. Dass der Kompetenzbegriff insbesondere von der empirischen Bildungsfor- 


\section{Didaktik subjektorientierten Lernens und Lehrens}

schung favorisiert wird, verstärkt die Vorbehalte ebenfalls - bis hin zu „Verschwörungstheorien“ mit „Strategien der Durchsetzung eines ökonomistischen Menschenbildes in Bildung und Bildungswesen" ist da alles vertreten (vgl. Krautz, 2012). Gerade in der Ernährungs- und Verbraucherbildung ist mit dem Begriff der Kompetenz (auch in der ubiquitären Weinert'schen Fassung) sowohl der Gegenstandsbereich als auch der erwünschte und gewollte Outcome präzise(r) zu beschreiben und in entsprechende Lernprozesse überzuführen; mit REVIS liegt diesbezüglich ein tragfähiges Konzept vor!

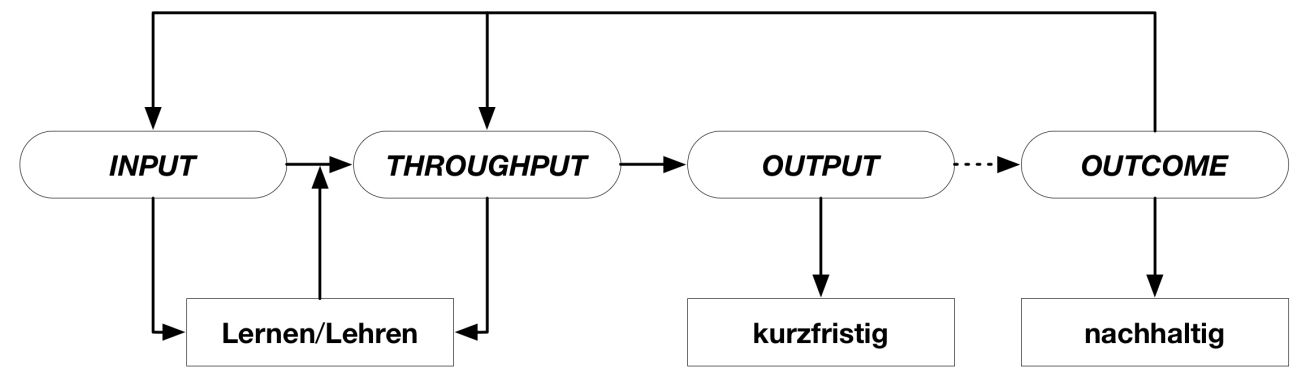

Abb. 5: Von Input zu Outcome - und vice versa (Quelle: Eigene Darstellung)

Das didaktische Denken erfährt insofern eine radikale Richtungsänderung, als nicht mehr ausschließlich Überlegungen zum Input am Beginn der didaktischen Überlegungen einschließlich der methodischen Throughput-Prozesse stehen und (und man am Output misst, was kurzfristig davon noch übriggeblieben ist), sondern vom gewünschten nachhaltigen Outcome her (quasi rückwärts) bestimmt, was als Input dafür tauglich ist und mit welchen Prozessen des Throughputs dieser auch erreicht werden kann; dass darin und dazwischen auch der Output überprüft und entsprechende Qualifikationen erteilt werden können, widerspricht der damit intendierten Kompetenzentwicklung nicht!

Mit Kompetenzen wird ja nicht einfach nur das Verfügen über fachspezifisches Wissen postuliert, sondern in der Formulierung entsprechender Fähigkeiten, Fertigkeiten und Bereitschaften zur Lösung von Aufgaben- und Problemstellungen wird sehr deutlich die Verklammerung von Wissen und Handeln (vgl. Brandl, 2013) - und nicht deren sukzessive Abfolge - thematisiert. Damit Wissen überhaupt handlungsrelevant werden kann - und als „träges Wissen“ dafür nicht zur Verfügung steht - ist bereits beim Erwerb sicherzustellen, dass nicht (ausschließlich) die Fachsystematik die Lerninhalte vorgibt, sondern darüber hinaus über authentische Bezüge und situiertes Lernen sichergestellt wird, dass (fach-)wissenschaftliche Konzepte der EVB in alltagsrelevante lebensweltliche Kontexte eingebunden werden. Der Gefahr, dass die Anbindung an Alltag und Lebenswelt lediglich eine motivationsauslösende und -fördernde Funktion im Rahmen des Unterrichts darstellt und dementsprechend „oberflächlich“ - weil allzu offensichtlich - gehandhabt wird, ist demgegenüber dafür Sorge zu tragen, dass Lernumgebungen „nicht nur konkrete Lehr-Lern- 


\section{Didaktik subjektorientierten Lernens und Lehrens}

arrangements umfassen, sondern als theoretische Voraussetzung auch fachdidaktisch durchdrungene Spezifizierungen und Strukturierungen von Lerngegenständen“ (Prediger et al., 2013, S. 11). Dies bedeutet

- fachliche Substantialität im Hinblick auf bildungswirksame Rekonstruktion des Gegenstandsbereichs,

- explizite unterrichtliche Anknüpfungen an Vorerfahrungen und Perspektiven der Lernenden,

- Berücksichtigung von Eigen- und Fremdperspektiven in der individuellen Sinnkonstruktion für die Strukturierung des Lerngegenstandes,

- Situierung in tragfähigen Kontexten, die zum einen Zugänglichkeit und zum anderen Transferierbarkeit ermöglichen.

\subsection{Lerntheoretische Grundlagen einer Didaktik subjektorientierten Lernens und Lehrens}

Traditionelle Lerntheorien sind nach Faulstich (2013) insofern zu einem vorläufigen Ende gekommen, als sie zwar auf die eigenen Aktivitäten und Konstruktionen durch die Individuen abstellen, allerdings den ,missing link“ zwischen Subjekt und Struktur noch schuldig blieben: „Alle Versuche, die Psyche aus der Psyche zu begreifen, drehen sich im Kreis. Erst wenn man das Ich-Welt-Verhältnis nicht mehr nur als psychisches Problem, sondern als Resultat eingreifender Praxis auffasst, durch Hinwendung zur Praxistheorie, kann der Zirkel durchbrochen werden“" (S. 13).

Der "theoretische Kern" eines Lernbegriffs, der nicht nur lerntheoretischen, sondern auch bildungstheoretischen Ansprüchen der „Resubjektivierung von Kultur“ (Plöger, 2011, S. 93) gerecht wird, besteht in folgenden Eckpunkten:

- „Lernen ist immer ein Neu-, Hinzu- und Umlernen, dessen Ergebnis in einer Struktur vorliegt.

- Lernen bringt das Strukturelle und Allgemeine des betreffenden Sachverhaltes zu Ausdruck.

- Lernen als Reduktion von Vielfalt und Komplexität bedeutet zwar Verlust an Konkretheit, dafür aber auch Gewinn an Klarheit und Einsicht in den Sachverhalt.

- Der Grad der notwendigen Reduktion von Vielfalt und Komplexität ist vom jeweiligen Lerngegenstand abhängig.

- Die durch Beziehungsstiftung gewonnenen Strukturen stehen ihrerseits immer im Zusammenhang mit vorangegangenen bzw. weiterführenden Beziehungsstiftungen“(Plöger, 2011, S. 92/93).

Unschwer zu erkennen ist im Kern die konstruktivistische Basistheorie von Piaget, nach der das Wissen und Können in Form sensorischer, begrifflicher und operatorischer Schemata vorliegen. Damit erfolgt auch die „Welterschließung“: Solange die 


\section{Didaktik subjektorientierten Lernens und Lehrens}

Anwendung vorhandener Schemata ausreicht, bestimmte Aufgaben zu bewältigen, besteht wenig Anlass, diese Schemata zu ändern. Piaget beschreibt diesen Prozess als Assimilation. Sollten diese Schemata zum Problemlösen (auf bislang unbekannten Terrain) allerdings nicht ausreichen, ist die Aneignung neuer Schemata notwendig. Diesen Prozess versieht Piaget mit dem Begriff der Akkomodation - eine Bezeichnung für den eigentlichen Lernprozess. Konkret: Mit den oft so genannten „Einführungsstunden“ ist immer eine Akkomodation gefordert, so genannte „Anwendungsund Übungsstunden“" hingegen sind „klassische“ Assimilationen. Über die stets notwendig werdende und stattfindende Adaptation erfolgt permanent die Anpassung der kognitiven Struktur an die Erfordernisse der Bewältigung von (Lern-)Aufgaben und Problemen (vgl. Brandl, 2012).

Fokus

Didaktik/

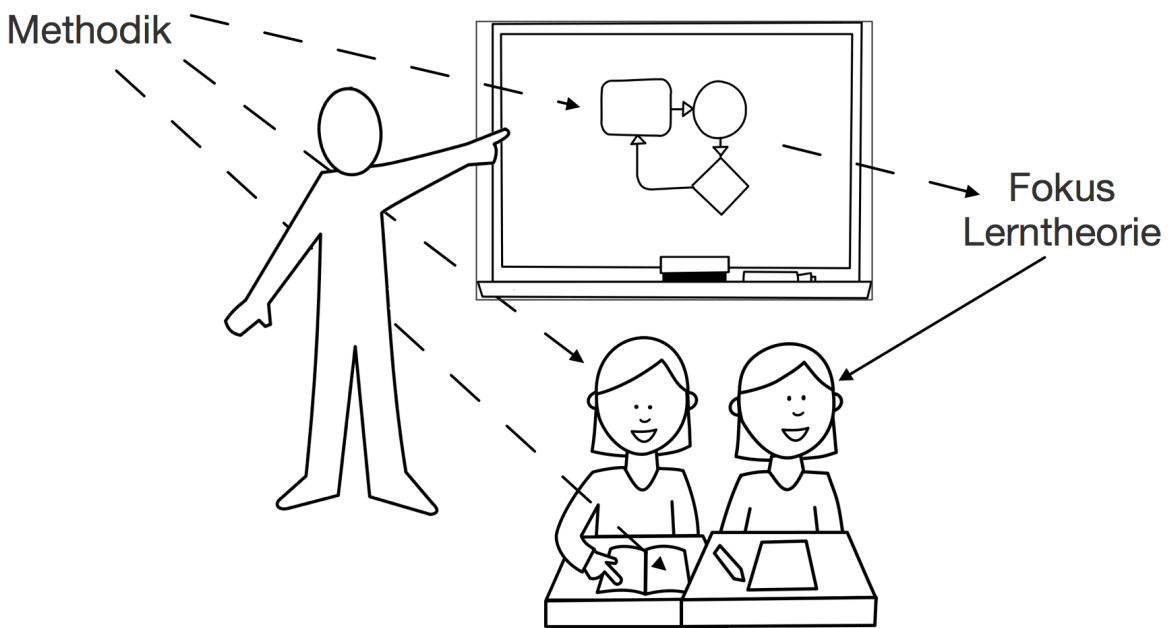

Abb. 6: Lernen im Fokus von Lerntheorie und (Fach-)Didaktik

Neben einer lerntheoretischen Fundierung bedarf subjektorientiertes Lernen und Lehren ebenfalls einer didaktischen Ausrichtung. Dabei ist „Bescheidenheit“ insofern angesagt, als „dass wir nicht so sehr ,neue' didaktische Theorien brauchen, die sich gegen andere abgrenzen und ihre selektive Leistung für das ,Ganze' ausgeben, sondern integrative Perspektiven, durch die die Anschlussfähigkeit zwischen Theorien und deren spezifischer Stellenwert sichtbar wird“" (Scholl, 2011, S. 39). Vielmehr ist das Augenmerk darauf zu richten, die „begriffliche und strukturelle Grundlagen für eine integriert(er)e didaktische Theorie des Unterrichts zu schaffen“" (Scholl, 2011, S. 39). Und eine dieser grundlegenden Begriffe ist Lernen: „Lernen ist für Schülerinnen und Schüler die zentrale Tätigkeit im Unterricht. Um so erstaunlicher ist es, dass in den ,großen' didaktischen Theorien das Thema Lernen mehr oder weniger ausgeblendet wird“ (Plöger, 2011, S. 85) - und auch in dezidiert fachdidak- 


\title{
Didaktik subjektorientierten Lernens und Lehrens
}

tischen Überlegungen gerät das Lernen über die Fokussierung auf die fachwissenschaftlich begründete Auswahl der Inhalte und Diskussion der Bedingungen der methodischen Unterrichtsgestaltung dann und wann ins Hintertreffen. „Diese Bedingungen sollten aber nicht mit dem Prozess des Lernens gleich gesetzt werden. Denn eine Entität, die eine andere bedingt, kann mit dieser anderen nicht identisch sein“ (Plöger, 2011, S. 85). Plöger (2011) moniert damit zu Recht, dass Lernen ein „blinder Fleck in Unterrichtstheorien“ ist, den man mit „einheimischen“ Mitteln aus dem Haus der Didaktik allerdings nicht beseitigen kann und das lerntheoretische Fundament deshalb ,aushäusig“ suchen und finden muss.

Nach Faulstich (2013) müssen sich Lerntheorien, insbesondere solche mit einer „praxeologischen, subjekt- und gleichzeitig kontextorientierten“ (S.19) Ausrichtung, „an den Anforderungen Konsistenz, Komplexität, Angemessenheit und Offenheit“ (S. 11) messen lassen. Eine angemessene Lerntheorie sollte demnach:

- begrifflich hinreichend geklärt und möglichst konsistent sein,

- hinreichende Komplexität aufweisen, welche den Gegenstand nicht vorschnell vereinfacht und ihn unangemessen begrenzt,

- gegenstandsadäquat sein: Eine Lerntheorie, welcher es um ein Erfassen menschlichen Lernend geht, ist einzubeziehen in Konzepte der gesellschaftlichen Tätigkeit bzw. des bewussten Handelns,

- sinnvollerweise durch Offenheit gekennzeichnet sein., dabei auch die bedingte Freiheit menschlichen Handelns berücksichtigen, bei dem Aneignung immer auf die je eigene Entscheidung zurückzuführen ist,

- immer auf konkrete Fälle menschlichen Lernens fokussieren. Diese zu interpretieren und auf ihren Kontext zu reflektieren, ist Aufgabe theoretischer Modelle und empirischer Konzepte menschlichen Lernens (Faulstich, 2013, S. 9/10).

\section{3 ...und Lehren vom Standpunkt des lernenden Subjekts aus denken}

\begin{abstract}
„Unter subjekttheoretischer Perspektive muss es auf der Lehrenden-Seite darum gehen, im Gegenüber die Hauptperson der Lehr-Lern-Zusammenhänge zu sehen. Das heißt Abschied zu nehmen von dem Missverständnis, Lehre würde automatisch Lernen zur Folge haben“ (Meueler, 2011, S. 976).
\end{abstract}

Jenseits der (Schein-)Gefechte über die Tragfähigkeit und Reichweite konstruktivistischer Theorien (vgl. Gruschka, 2011) und „unabhängig davon, ob sie sich radikal, strikt, gemäßigt, kommunikativ oder realistisch verstehen - alle Protagonisten betonen in der Regel (und das ist wohl der kleinste gemeinsame Nenner aller konstruktionistischen wie konstruktivistischen Positionen), dass Menschen bei ihrem Handeln, aber auch bei dem selektiven Auf- und Ausbau von 


\section{Didaktik subjektorientierten Lernens und Lehrens}

Wissen und auch bei der Wahrnehmung immer und unhintergehbar von den Normen und Werten der jeweiligen Gesellschaft, von den Konventionen ihrer Gruppe und natürlich von den aktuellen Machtverhältnissen geleitet, geführt oder gar gesteuert werden und dass all dies mittels Kommunikation geschieht" (Reichertz, 2013, S.62).

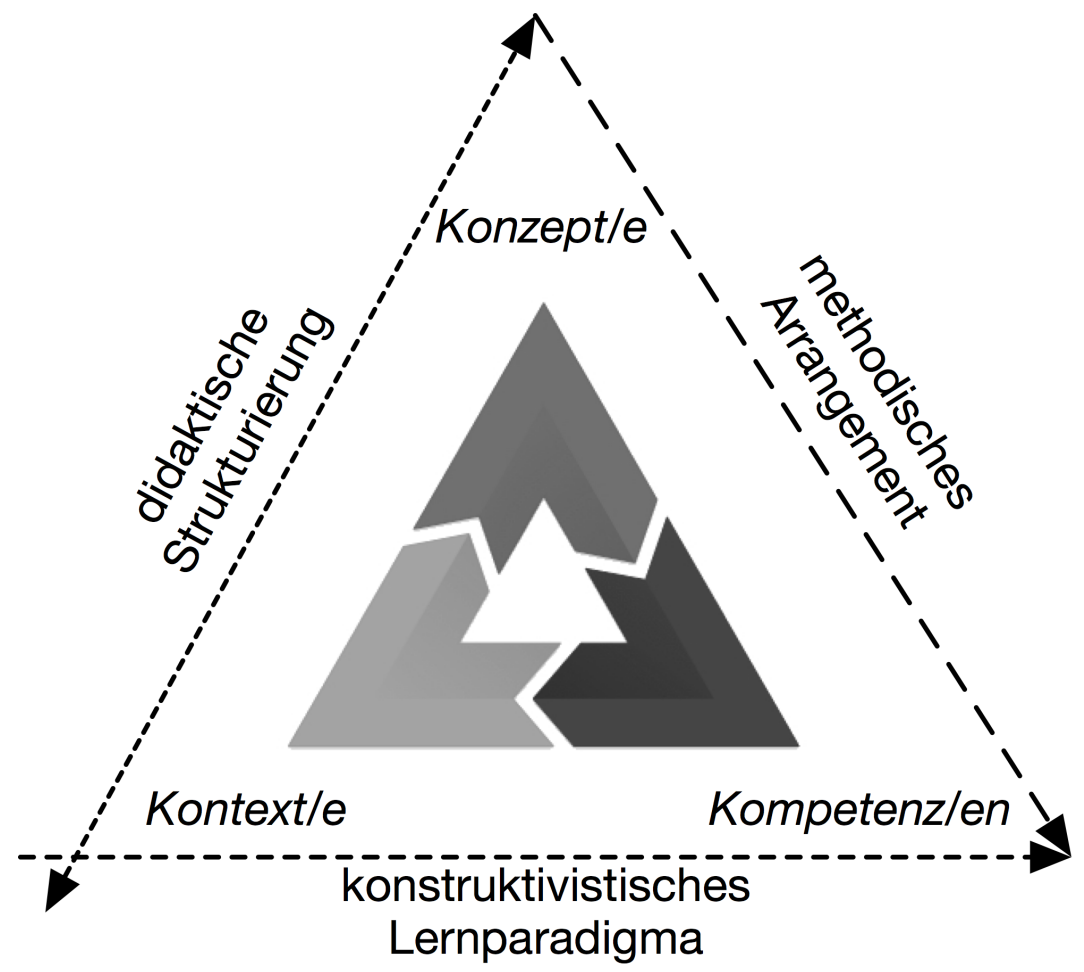

Abb. 7: Konstruktivismus - Kontext/e - Konzept/e - Kompetenz/en

\subsection{Konstruktivistisches Lernparadigma}

In Bezug auf ein konstruktivistisches Lernparadigma und der darauf aufbauenden Konzeptionen konstruktivistischer Didaktik(en), ist ein Ertrag festzuhalten, nämlich, „dass der Konstruktivismus einerseits eine faszinierende Denkschule ist, die viele Anregungen für den Pädagogen bereithält, dass er andererseits aber nicht als Grundlagentheorie für die Pädagogik ausreicht“ (de Haan \& Rülcker, 2009, S. 189). Die Sicht auf die Lernenden und Lern-Lehrprozesse ist damit geschärft und die „Denkrichtung" geändert worden, auch wenn die Quintessenz eigentlich recht banal daherkommen mag: „Lernen kann jeder nur für sich allein; aber Vieles, was man sich aneignen will, kann man nur mit Hilfe anderer erlernen“ (Plöger, 2011, S. 86). Geschärft wurde mit konstruktivistischen Denkmustern generell der Fokus auf das Lernen und - daraus folgernd - auf den Weg zu einer Theorie „lernseits“" von Unterricht (Schratz, 2009) das „Lehren im Modus des Lernens“ zu denken. 


\section{Didaktik subjektorientierten Lernens und Lehrens}

Konstruktivistischen Ansätzen ist nach Tulodziecki et al. (2009) eine (mehr oder weniger stark ausgeprägte ähnliche) Sicht auf Unterricht gemein:

Unterricht sollte demnach

- „den Lernenden Möglichkeiten eröffnen, individuelle Vorstellungen, Sinn- und Bedeutungskonstruktionen in den Lehr-Lernprozess einzubringen und sich mit eigenen und den Konstruktionen anderer in vergleichender Weise auseinander zu setzen.

- individuelle Vorstellungen $\mathrm{zu}$ bestimmten Wirklichkeitsbereichen mit neuen Erfahrungen zu kontrastieren und auf ihre ,Viabilität' hin zu prüfen.

- Lerngegenstände aus unterschiedlichen Perspektiven mit unterschiedlichen Methoden zu erschließen und in ihrer jeweiligen Vorläufigkeit und Situationsgebundenheit zu erschließen.

- in seiner Planung und Durchführung als ein reflexiver Prozess aufgefasst werden, der zu einer variantenreichen und situationsangemessenen Gestaltung und Auswertung von Lehr- und Lernprozessen führt und damit auch auf eine ständige Qualitätsverbesserung ausgerichtet ist“" (S. 257).

\subsection{Subjektorientierte Unterrichtskonzeption: Lehren im Modus des Lernens denken}

Die (zunehmend) feststellbare sprachliche Kennzeichnung in der Reihenfolge Lernen-Lehren (und z. B. nicht mehr Lehr-Lern-Forschung) ist nicht willkürlich (wie es scheinen mag), sondern verdankt sich einem (nur sprachlich marginalen) Perspektivwechsel: „Lehre weist mögliche sinnvolle Lernwege durch die Berge des Wissens. Gehen müssen die Pfade aber die Lernenden selbst. Es geht um Vermitteln von Lerninteressen und Lernproblematiken“ (Faulstich, 2013, S. 18).

Kernelemente einer subjektorientierten Unterrichtskonzeption für die Ernährungs- und Verbraucherbildung sind (vgl. Brandl, 2008, S. 6) eine

- dezidiert subjektbezogene Sichtweise, die sich

○ an der Lebenswelt,

○ den Erfahrungen und Gestaltungsmöglichkeiten im Alltag und

○ am Status und dem Potenzial der biografischen Entwicklung orientiert

- kontextorientierte Anbindung, die

- die subjektive Relevanz von Lernprozessen und -resultaten

- mit fachsystematischen Erfordernissen verknüpft und

- Strukturen für Handlungsoptionen aufweist

- $\quad$ eine handlungsorientierte Ausrichtung, die 


\section{Didaktik subjektorientierten Lernens und Lehrens}

- auf der Basis von Selbstwirksamkeitsüberzeugungen und Selbststeuerung/-regulation

○ die Möglichkeit zur persönlichen Entwicklung anbietet

und ein „konsequentes Bemühen um die Förderung nachhaltigen Lernens“ (Beyer, 2014, S.299) nach folgenden zentralen Prinzipien (Beyer, 2014, S. 300-303):

- $\quad$ Sicherstellung

○ der Bedeutsamkeit des zu Lernenden

o der erforderlichen Lernvoraussetzungen

- Einbettung des Lernstoffs in Aufgaben, Fragen oder Problemen

- Förderung

○ kumulativen Lernens

○ kooperativen Lernens

○ kategorial strukturierten Lernens

$\circ$ anwendungsbezogenen Lernens

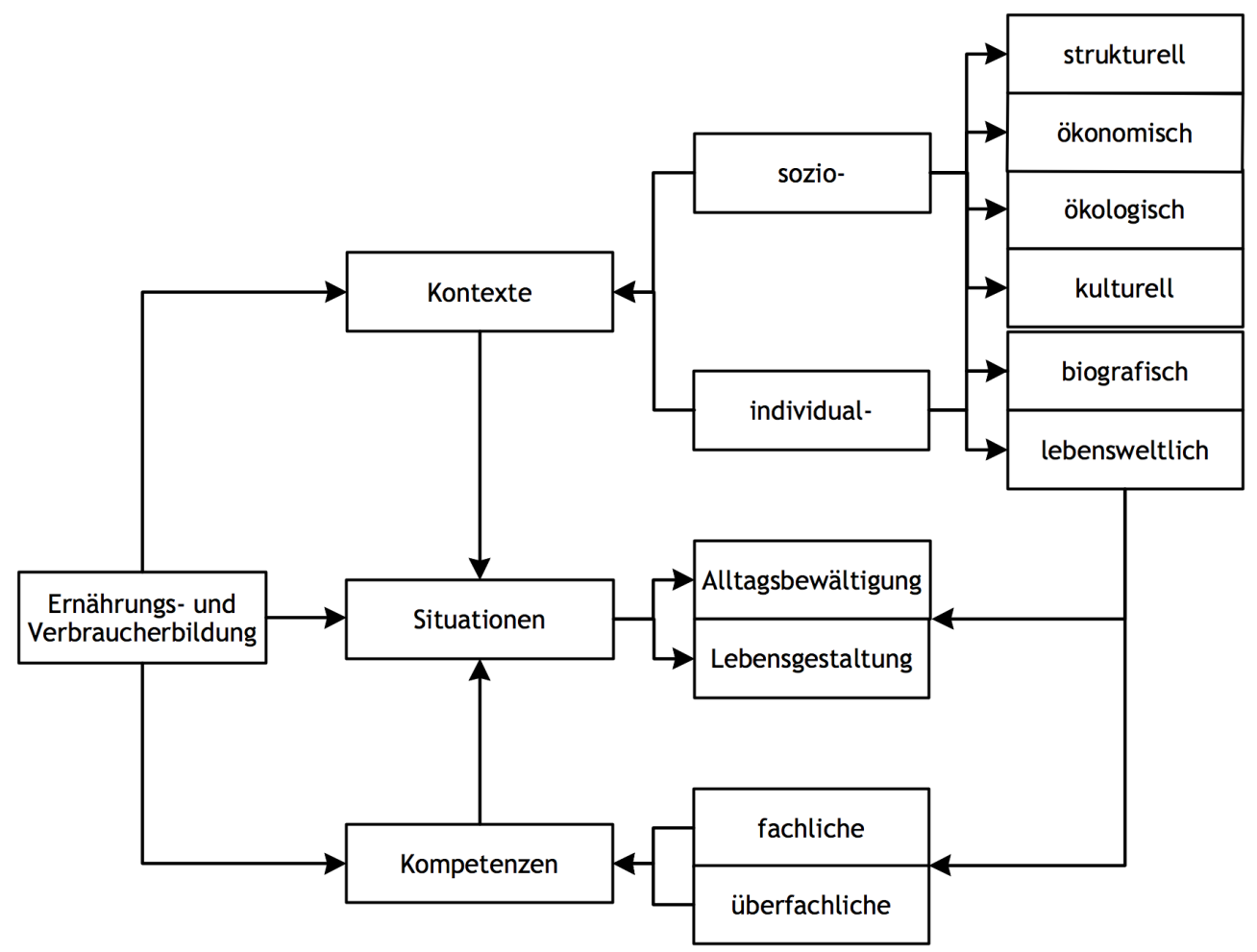

Abb. 8: Kontexte - Situationen - Kompetenzen (Quelle: Eigene Darstellung) 


\section{Didaktik subjektorientierten Lernens und Lehrens}

\subsection{Didaktische Reflexion...}

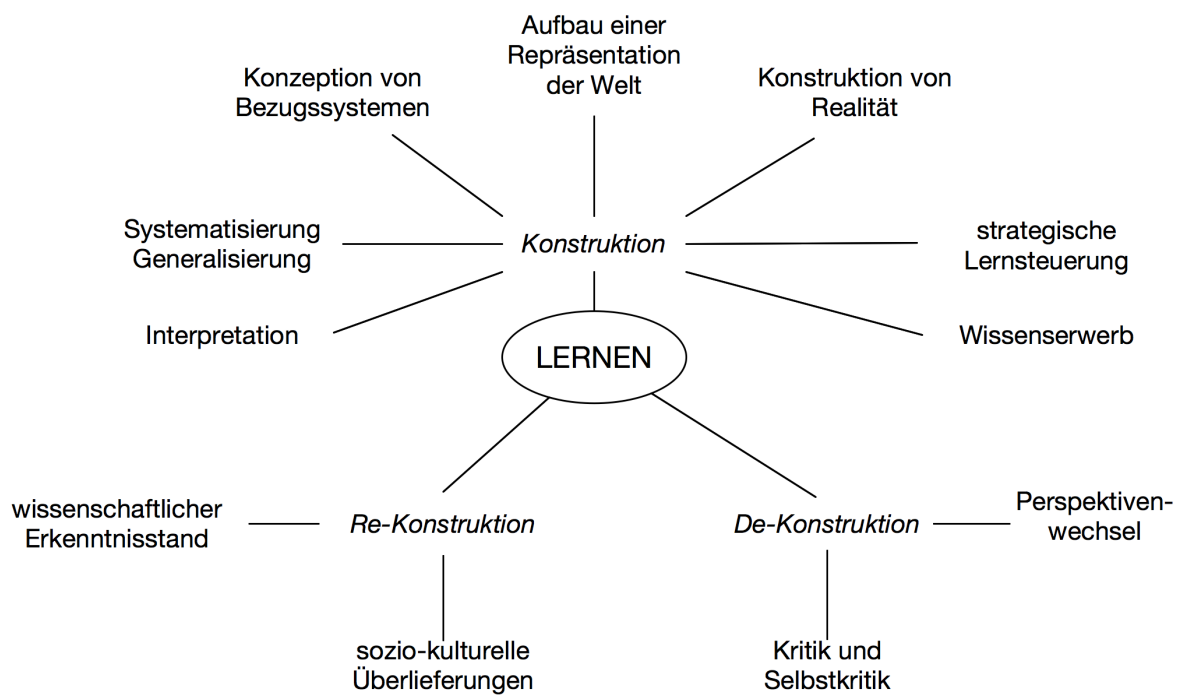

Abb. 9: Konstruktivistisches Lernen (Quelle: nach Andersen, 2010, S. 11; verändert)

Folgt man den griffigen Vorstellungen einer konstruktivistischen Didaktik nach Reich (2012), sind - zunächst unabhängig von bestimmten Fächern und Gegenständen - prinzipiell drei Zugänge für eine konstruktivistische Unterrichtsgestaltung denkbar:

- Konstruktion: Nach einem konstruktivistischen (auch neurobiologischen) Grundverständnis ist jegliche Wissensaneignung eine Konstruktionsleistung der lernenden Subjekte. Die Lerninhalte können nur verstanden werden, wenn sie in die vorhandenen Schemata eingebunden und mit Sinn verbunden werden können. Ein unmittelbarer Transfer des Wissens vom Lehrenden zum Lernenden ist somit ausgeschlossen; Lehrende können lediglich (aber das durchaus) Angebote in entsprechenden Lernumgebungen machen.

- Re-Konstruktion: Nicht alle Lerninhalte sind von den Lernenden immer wieder und absolut neu in allen Facetten selbst zu erarbeiten; über die gedanklich strukturelle Kopplung und instruktionale Kommunikation der Lehrenden mit den Lernenden ist ein re-konstruierender Nachvollzug vorhandenen Wissens möglich.

- De-Konstruktion: Weder die Lerninhalte selbst, noch die Zugänge zu diesen sind unveränderbar festgeschrieben: Unterschiedliche Blickwinkel, Perspektiven, auch Zweifel und Kritik verändern gleichermaßen die 


\section{Didaktik subjektorientierten Lernens und Lehrens}

Sache und den Betrachter: Im Gegensatz zum „Mainstream“ lassen sich Gegenstände, Personen, Situationen, Prozesse etc. auch (ganz) anders betrachten und behandeln.

Konstruktion, Re-Konstruktion und De-Konstruktion können als Folie einer didaktischen Reflexion zur Aufgabenstellung sowohl für Lernende als auch Lehrende dienen:

Tab. 1: Didaktische Reflexionstafel (Quelle: Eigene Darstellung nach Reich, 2012, S. 182; verändert und ergänzt)

\begin{tabular}{l|c|c|c}
\cline { 2 - 4 } & Prozess/e & Methode/n & Resultat/e \\
\hline $\begin{array}{l}\text { De- } \\
\text { Konstruktion }\end{array}$ & Enttarnen/lassen & Bezweifeln/lassen & Verständnis \\
\hline Konstruktion & Erfinden/lassen & Begründen/lassen & Erkenntnis \\
\hline $\begin{array}{l}\text { Re- } \\
\text { Konstruktion }\end{array}$ & Entdecken/lassen & Verallgemeinern/lassen & Kenntnis \\
\hline
\end{tabular}

\section{4 ...und „Didaktische Rekonstruktion“}

Für die didaktische Strukturierung des Gegenstandsbereiches der EVB bietet sich das Modell der „Didaktischen Rekonstruktion“ (Kattmann et al., 1997; Kattmann, 2007; Duit et al. 2012; Gropengießer \& Kattmann, 2013) - obwohl als „Rahmen für naturwissenschaftsdidaktische Forschung und Entwicklung“" entwickelt - insofern geradezu auch für die EVB an, als mit der kennzeichnenden Wechselwirkung von fachlichen, didaktischen sowie subjektbezogenen Aspekten die komplexen (fach-) wissenschaftlichen Inhalte nicht 1:1 und damit unverändert in den schulischen Fachunterricht übernommen werden können, sondern in überfachliche Bezüge interdisziplinär und der Berücksichtigung sozialer, kultureller und individueller Zusammenhänge eingebettet werden müssen.

Die Didaktische Rekonstruktion ist „eine praktische Theorie“ (Kattmann, 2007): „Damit werden die wesentlichen Teile fachdidaktischer Arbeiten explizit gemacht, systematisch aufeinander bezogen und für die Praxis relevant. Dadurch sollen die im Rahmen der Didaktischen Rekonstruktion durchgeführten Forschungsarbeiten lernförderlicher werden als solche, die sich allein auf eine fachwissenschaftliche Sachstruktur oder lernpsychologische Prinzipien stützen könnten“ (Kattmann, 2007, 94). 


\section{Didaktik subjektorientierten Lernens und Lehrens}

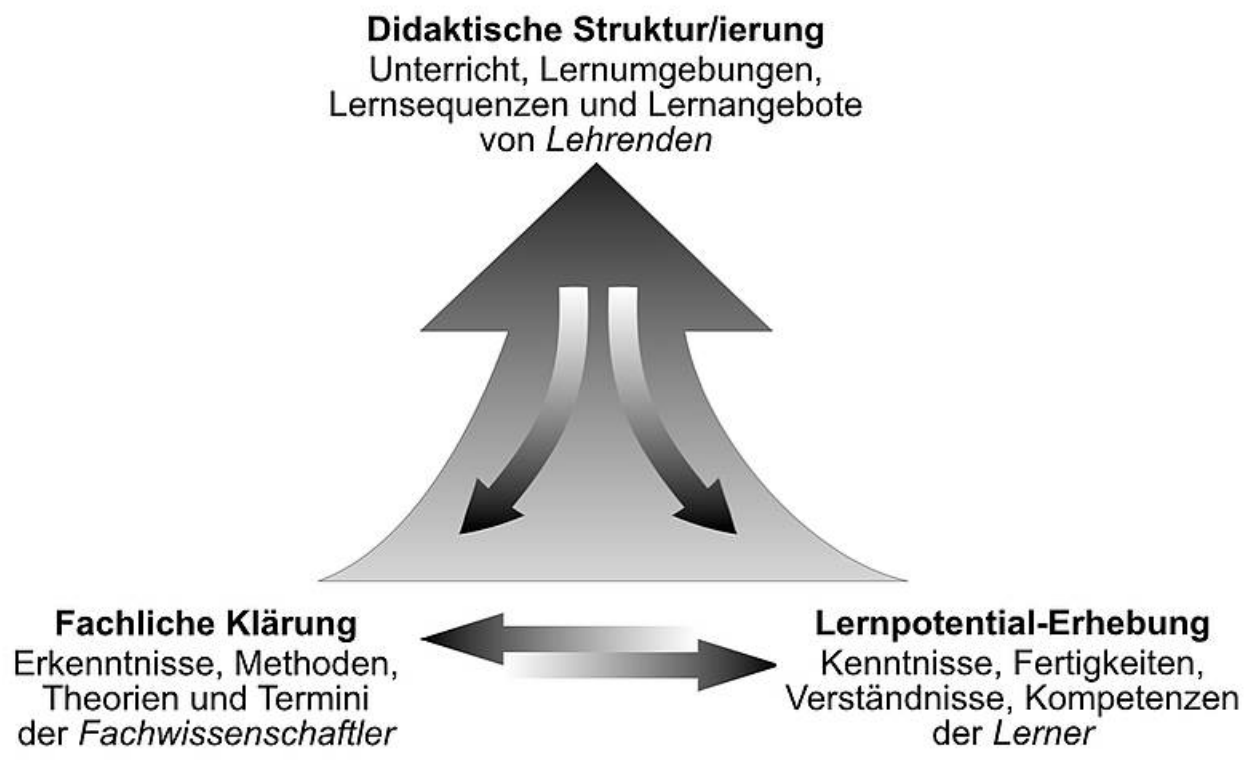

Abb. 9: Das Modell der Didaktischen Rekonstruktion (Quelle: mit Genehmigung aus Gropengießer \& Kattmann, 2013)

Sie versteht sich als eine Metatheorie, die sich mehrerer Teiltheorien bedient und $u$. a. auf konstruktivistische Theorien vom Lernen und Lehren, auf die Theorie vom erfahrungsbasierten Verstehen und Theorien zur Vorstellungsbildung und -änderung (Kattmann, 2007, S. 97) stützt, diese zusammenführt, modifiziert und nutzbar macht. „Man mag die Didaktische Rekonstruktion wegen des bloßen Zusammenbringens von Theorien nicht selbst als Theorie betrachten. Dies wäre jedoch verfehlt, da durch das Zusammenfügen der Charakter der Teil-Theorien verändert und eine neue Qualität erreicht wird“" (Kattmann, 2007, S. 97/98).

\section{Adaptive Lehrkompetenz und Lernumgebungen}

„Lerntheorie braucht ein angemessenes Konzept von Lehre“ (Faulstich, 2013, S. 18) - und auf der personalen Seite eine entsprechend elaborierte Lehrkompetenz!

Ausgangspunkt der Überlegungen zur Konzeption des Konstrukts ,adaptive Lehrkompetenz" ist nach Brühwiler (2014) die ausgeprägte Individualität der Schülerinnen und Schüler - was die Persönlichkeitsmerkmale und Lernvoraussetzungen im Allgemeinen anbelangt - und die daraus resultierende Heterogenität von Schulklassen, die die Lehrkräfte in sehr anspruchsvoller Weise herausfordern: „Sie sollen nicht lediglich Stoff an eine Klasse vermitteln, sondern ihr unterrichtliches Handeln auf die unterschiedlichen individuellen Lernbedürfnisse der einzelnen Schülerinnen 


\section{Didaktik subjektorientierten Lernens und Lehrens}

und Schüler ausrichten, um so deren Lernen bestmöglich zu unterstützen“" (Brühwiler, 2014, S. 11). Adaptive Lehrkompetenz als adaptive Planungs- und Handlungskompetenz gefasst (Beck et al., 2008; Brühwiler, 2014), bezeichnet die Fähigkeit, Planung und Durchführung des Unterrichts auf die Heterogenität und Individualität der Schülerinnen und Schüler situationsspezifisch so auszurichten, dass für möglichst viele Schülerinnen und Schüler bestmögliche Bedingungen für die Kompetenzentwicklung bestehen. „Adaptive Lehrkompetenz bedeutet in didaktischer Hinsicht eine empiriegestützte Beurteilung von unterschiedlichen Unterrichtsverfahren und ihre darauf abgestützte adaptive Verwendung im Unterricht, je nach den vorhandenen Bedingungen und den angestrebten Zielen" (Beck et al., 2008, S. 46)

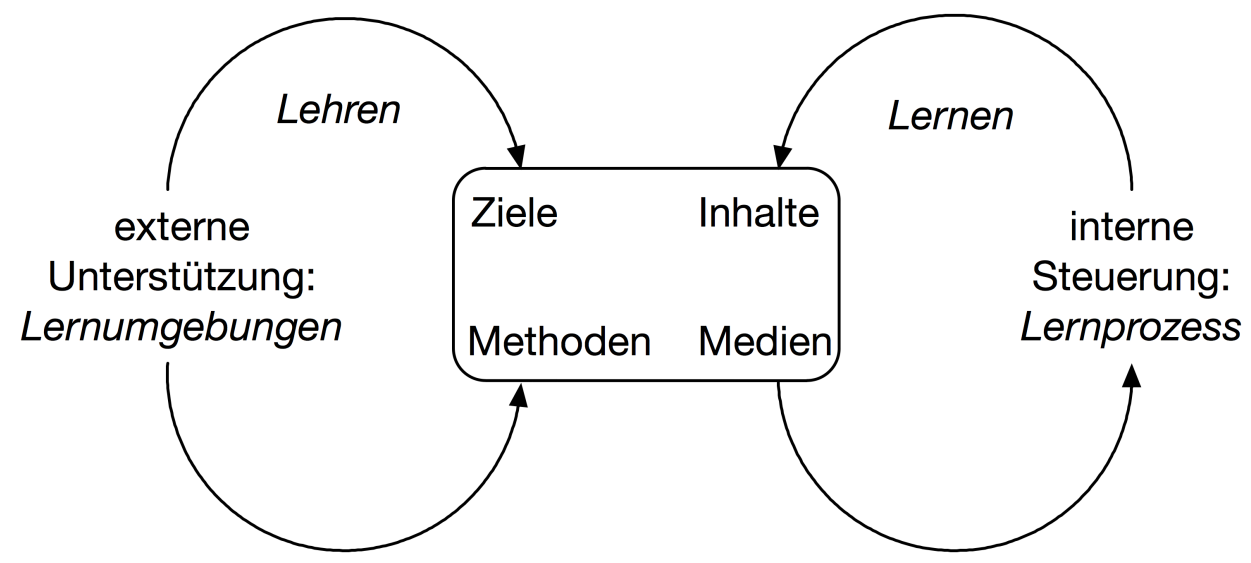

Abb. 10: Adaptivität: Lehren und Lernen (Quelle: Eigene Darstellung)

Die Unterrichtsprinzipien Differenzierung und Individualisierung sind in allen didaktischen Modellen unbestritten, mit der adaptiven Lehrkompetenz „liegt ein kognitivkonstruktivistisches Verständnis von Lernen zugrunde“ (Beck et al, 2008, S 39), das eine deutliche Trennung zwischen der je individuellen Steuerung des Lernens durch die Lernenden und den (prinzipiell beschränkten) Einfluss, den Lehrende auf diesen Lernprozess nehmen können. Mit der Gestaltung von Lernumgebungen kann lediglich - aber dies durchaus - eine entsprechende Unterstützung erfolgen.

Dieses setzt voraus, dass die Lehrenden nach Beck et. al. (2008, S. 41/42) (zumindest) über die folgenden Teilkompetenzen verfügen:

- Sachkompetenz: reichhaltiges, flexibel nutzbares Sachwissen, in dem sich die Lehrperson leicht und rasch geistig bewegen kann;

- diagnostische Kompetenz: die Lernenden bezüglich ihrer Lernvoraussetzungen und -bedingungen (Vorwissen, Lernweisen, Lerntempo, Lernschwächen) und bezogen auf den jeweiligen Unterrichtsgegenstand sowie ihrer Lernergebnisse zutreffend einschätzen können; 


\section{Didaktik subjektorientierten Lernens und Lehrens}

- didaktische Kompetenz: reichhaltiges methodisch-didaktisches Wissen und Können einschließlich der Kenntnisse der Vor- und Nachteile und der Erfolg versprechenden einsetzbaren didaktischen Möglichkeiten

- Klassenführungskompetenz: die Klasse so zu führen, dass sich die Lernenden aktiv, anhaltend und ohne zu viel störende Nebenaktivitäten mit dem Unterrichtsgegenstand als Grundlage für Lernfortschritt und Lernerfolg auseinandersetzen können.

\section{Zwischenstand und Wiederanstoß}

Die Vielfalt, mit der ein Subjektstandpunkt und/oder die Subjektorientierung zur Begründung pädagogisch/psychologisch/didaktisch/methodisch interessanter Momente des Lernens und Lehrens fungieren, liegt sowohl in der offenen begrifflichen Fassung von „Subjekt“, als auch in derjenigen ihrer differenten kategorialen Verortung in diversen „didaktischen Häusern“ - eine „Subjektorientierte Didaktik“ in der EVB muss sich der Klärung dieser Frage- und Problemstellung stellen. Bartsch (2012) hat dieses Desiderat angesprochen: „Erkenntnisse aus der Lernpsychologie und der allgemeinen Pädagogik [sind] hilfreich, dennoch ersetzen sie keine fachdidaktische Forschung, deren Weiterentwicklung bezüglich der Subjektorientierung in der Ernährungs- und Verbraucherbildung sicherlich lohnenswert wäre“ (S. 62)!

\section{Anmerkung}

1 „Moderne“ pädagogische Anthropologie differenziert diesen Ausgangspunkt:

Der Mensch ist demnach ein Wesen, das als Mensch gleichzeitig

- $\quad$ erzogen wird und selbst erzieht;

- lernfähig und lernbedürftig ist;

- bildungsfähig und bildungsbedürftig ist,

- lehrend und unterrichtend ist;

- $\quad$ sich sozialisiert und kultiviert (vgl. Wulf \& Zirfas, 2014, S. 14).

\section{Literatur}

Andersen, K.N. (2010). Methodenpool Grundschule. Unterricht konstruktivistisch gestalten. Weinheim: Beltz.

Bartsch, S. (2008). Subjektorientierung in der Ernährungs- und Gesundheitsbildung. Ernährung - Wissenschaft und Praxis, 2(3), 100-106.

[DOI: $10.1007 / \mathrm{s} 12082-008-0152-5]$.

Bartsch, S. (2012). Subjektorientierung. Ein Beitrag zur kompetenzorientierten Aufgabengestaltung in der Verbraucherbildung. Haushalt in Bildung \& Forschung, 2(3). 52-64. 


\section{Didaktik subjektorientierten Lernens und Lehrens}

Beck, E., Baer, M., Guldimann, T., Bischoff, S., Brühwiler, P., Müller, P., Niedermann, R., Rogalla, M. \& Voigt, F. (2008). Adaptive Lehrkompetenz. Analyse und Struktur, Veränderung und Wirkung handlungssteuernden Lehrerwissens. Münster: Waxmann.

Bengel, J., Strittmatter, R. \& Willmann, H. (2001). Was erhält Menschen gesund? Antonovskys Modell der Salutogenese - Diskussionsstand und Stellenwert (Forschung und Praxis der Gesundheitsförderung, Bd.6, erw. Neuaufl.). Köln: BZgA. [www.bzga.de/botmed_60606000.html.

Beyer, K. (2014). Didaktische Prinzipien. Eckpfeiler guten Unterrichts. Baltmannsweiler: Schneider Verlag.

Bräuer, H. (2003). Person. In W.D. Rehfus (Hrsg.), Handwörterbuch Philosophie. Göttingen: Vandenhoeck \& Ruprecht. [www.philosophie-woerterbuch.de].

Brandl, W. (2008). Unterricht/en in der Ernährungs- und Verbraucherbildung. Haushalt \& Bildung, 85(3), 3-20.

Brandl, W. (2012). Begriffe - Konzepte - Argumente: Bausteine für mentale Konstruktionsprozesse im kompetenzorientierten Unterricht. Haushalt in Bildung \& Forschung, 1(3), 31-51.

Brandl, W. (2013). Wissen und Handeln: Diesseits und jenseits des ,Rubikon'. Haushalt in Bildung \& Forschung, 2(3), 3-20.

Brühwiler, C. (2014). Adaptive Lehrkompetenz und schulisches Lernen: Effekte handlungssteuernder Kognitionen von Lehrpersonen auf Unterrichtsprozesse und Lernergebnisse der Schülerinnen und Schüler. Münster: Waxmann.

Deci, E.L. \& Ryan, R.M. (2000). The „What“ and „Why“ of goal pursuits. Human needs and the self-determiation of behavior. Psychological Inquiry, 11(4), 227268. [DOI:10.1207/S15327965PLI1104_01].

de Haan, G. \& Rülcker, T. (2009). Der Konstruktivismus als Grundlage für die Pädagogik. Frankfurt/Main: Peter Lang.

Duit, R., Gropengießer, H., Kattmann, U., Komorek, M. \& Parchmann, I. (2012). The Model of Educational Reconstruction - A Framework for Improving Teaching and Learning Science. In Jorde, D. \& Dillon, J. (eds.), Science Education Research and Practice in Europe (S. 13-37). Rotterdam: Sense Publishers.

Faulstich, P. (2013). Menschliches Lernen. Eine kritisch-pragmatische Lerntheorie. Bielefeld: transcript.

Gropengießer, H. \& Kattmann, U. (2013). Didaktische Rekonstruktion. In H. Gropengießer, U. Harms \& U. Kattmann (Hrsg.), Fachdidaktik Biologie (9. Aufl., S. 16-23). Hallbergmoos: Aulis Verlag.

Gruschka, A. (2011). Verstehen lehren. Ein Plädoyer für guten Unterricht. Stuttgart: Reclam.

Kant, I. (1798). Anthropologie in pragmatischer Hinsicht.

[www.korpora.org/kant/aa07/324.html]. 


\section{Didaktik subjektorientierten Lernens und Lehrens}

Kattmann, U. (2007). Didaktische Rekonstruktion - eine praktische Theorie. In D. Krüger \& H. Vogt (Hrsg.), Theorien in der biologiedidaktischen Forschung (S. 93-104). Berlin: Springer.

Kattman, U., Duit, R., Gropengießer, H. \& Komorek (1997). Das Modell der Didaktischen Rekonstruktion - Ein Rahmen für naturwissenschaftsdidaktische Forschung und Entwicklung. Zeitschrift für Didaktik der Naturwissenschaften, 3(3), 3-18.

Keupp, H. (2012). Subjektgenese, Enkulturation und Identität. In H. Bockhorst, V.-I. Reinwand \& W. Zacharias (Hrsg.), Handbuch Kulturelle Bildung (S.98-102). München: kopaed.

[www.kubi-online.de/artikel/subjektgenese-enkulturation-identitaet].

Krapp, A. (2004). Individuelle Lebensinteressen als lerntheoretische Grundkategorie im Spiegel der pädagogisch-psychologischen Interessentheorie. In P. Faulstich \& J. Ludwig (Hrsg.), Expansives Lernen (S. 275-287). Baltmannsweiler: Schneider Verlag.

Krautz, J. (2012). Bildungsreform und Propaganda. Strategien der Durchsetzung eines ökonomistischen Menschenbildes in Bildung und Bildungswesen. In U. Frost \& M. Rieger-Ladich (Hrsg.), Demokratie setzt aus. Gegen die sanfte Liquidation einer politischen Lebensform (Sonderheft zur Vierteljahrsschrift für wissenschaftliche Pädagogik, S. 119-161). Paderborn: Schöningh.

Ludwig, J. (2005). Modelle subjektorientierter Didaktik. REPORT, 28(1), 75-80. [www.die-bonn.de/doks/ludwig0501.pdf].

Ludwig, J. \& Rihm, T. (2013). Der Subjektstandpunkt in der Didaktik. Jahrbuch für Allgemeine Didaktik (JfAD), 3, 83-96.

Lüdtke, N. \& Matsuzaki, H. (Hrsg.). (2011). Akteur - Individuum - Subjekt. Fragen zu "Personalität" und ,Sozialität". Wiesbaden: SpringerVS.

Methfessel, B. (2007). Salutogenese - ein Modell fordert zum Umdenken heraus Teil 1 - Antonovskys Modell der Salutogenese. Ernährungsumschau, 55(1), 704-709.

Methfessel, B. (2008). Salutogenese - ein Modell fordert zum Umdenken heraus Teil 2 - Herausforderungen für die Gesundheitsförderung. Ernährungsumschau, 55(1), 37-43.

Methfessel, B. \& Schlegel-Matthies, K. (2014). Alltagskultur: viel beschworen, wenig wissenschaftlich durchdrungen?! Hauswirtschaft und Wissenschaft, 62(1), 28-37.

Meueler, E. (2011). Didaktik der Erwachsenenbildung - Weiterbildung als offenes Projekt. In R. Tippelt \& A.v. Hippel (Hrsg.), Handbuch Erwachsenenbildung/Weiterbildung (5. Aufl., S. 973-987). Wiesbaden: VS Verlag.

Muckenfuß, H. (2004). Themen und Kontexte als Strukturelemente des naturwissenschaftlichen Unterrichts. Zu den Schwierigkeiten, systematisches Physiklernen zu organisieren. Physik und Didaktik in Schule und Hochschule, 3(2), 57-66. [http://phydid.physik.fu-berlin.de/index.php/phydid/article/view/23/23]. 


\section{Didaktik subjektorientierten Lernens und Lehrens}

Plöger, W. (2011). Lernen - ein blinder Fleck in Unterrichtstheorien?! In W. Meseth, M. Proske \& F.-O. Radtke (Hrsg.) (2011), Unterrichtstheorien in Forschung und Lehre (S. 85-102). Bad Heilbrunn: Klinkhardt.

Prediger, S., Komorek, M., Fischer, A., Hinz, R., Hußmann, S., Noschner, B. Ralle, B. \& Thiele, J. (2013). Der lange Weg zum Unterrichtsdesign. Zur Begründung und Umsetzung fachdidaktischer Forschungs- und Entwicklungsprogramme. In M. Komorek \& S. Prediger, S (Hrsg.), Der lange Weg zum Unterrichtsdesign. Zur Begründung und Umsetzung fachdidaktischer Forschungs- und Entwicklungsprogramme (S. 9-23). Münster: Waxmann.

Reich, K. (2012). Konstruktivistische Didaktik. Das Lehr- und Studienbuch mit Online-Methodenpool (5. Aufl.). Weinheim: Beltz.

Reichertz, J. (2013). Grundzüge des Kommunikativen Konstruktivismus. In R. Keller, H. Knoblauch \& J. Reichertz (Hrsg), Kommunikativer Konstruktivismus. Theoretische und empirische Arbeiten zu einem neuen wissenssoziologischen Ansatz (S. 49-68). Wiesbaden: SpringerVS.

Scholl, D. (2011). Der Unterrichtsbegriff in allgemeindidaktischen Theorien. In W. Meseth, M. Proske \& F.-O. Radtke (Hrsg.) (2011), Unterrichtstheorien in Forschung und Lehre (S. 37-49). Bad Heilbrunn: Klinkhardt.

Schratz, M. (2009). "Lernseits" von Unterricht. Alte Muster, neue Lebenswelten was für Schulen? Lernende Schule, 12, 16-21.

Terhart, E. (2013). Neuansätze in der Allgemeinen Didaktik: Ein Kommentar. Jahrbuch für Allgemeine Didaktik (JfAD), 3, 219-228.

Tulodziecki, G. (2002). Handlungsorientiertes Lernen zwischen Subjekt- und Sachorientierung. In B. Herzig \& U. Schwerdt (Hrsg.), Subjekt- oder Sachorientierung in der Didaktik? Aktuelle Beiträge zu einem didaktischen Grundproblem (S. 79-105). Münster: LIT-Verlag.

Tulodziecki, G., Herzig, B. \& Blömeke, S (2009). Gestaltung von Unterricht. Eine Einführung in die Didaktik (2. Aufl.). KlinkhardtUTB.

Wulf, C. \& Zirfas, J. (2014). Homo educandus. Eine Einleitung in die Pädagogische Anthropologie. In C. Wulf \& J. Zirfas. (Hrsg.), Handbuch Pädagogische Anthropologie (S. 9-27). Wiesbaden: SpringerVS.

\section{Verfasser}

Werner Brandl M.A.

Institutsrektor

Staatsinstitut für die Ausbildung von Fachlehrern

- Abteilung II -

Am Stadtpark 20

D-81243 München 